SIT-HEP/TM-53

\title{
Evolution of the curvature perturbations during warm inflation
}

\author{
Tomohiro Matsuda 1 \\ Laboratory of Physics, Saitama Institute of Technology, \\ Fusaiji, Okabe-machi, Saitama 369-0293, Japan
}

\begin{abstract}
This paper considers warm inflation as an interesting application of multifield inflation. Delta-N formalism is used for the calculation of the evolution of the curvature perturbations during warm inflation. Although the perturbations considered in this paper are decaying after the horizon exit, the corrections to the curvature perturbations sourced by these perturbations can remain and dominate the curvature perturbations at large scales. In addition to the typical evolution of the curvature perturbations, inhomogeneous diffusion rate is considered for warm inflation, which may lead to significant non-Gaussianity of the spectrum.
\end{abstract}

\footnotetext{
${ }^{1}$ matsuda@sit.ac.jp
} 


\section{Introduction}

Inflation is the most successful model for the large scale structure of the Universe in terms of the very early Universe. An inflationary Universe is consistent with current observations of the temperature anisotropy of the cosmic microwave background (CMB). Inflationary dynamics may be categorized in two ways: the original (supercooled) inflation scenario, and warm inflation [1]. In the warm inflation scenario, dissipative effects are significant and radiation production occurs concurrently during the inflationary period [2]. Basically, the spectrum of the cosmological perturbations created during inflation is expected to be scale-invariant and Gaussian. However, there are anomalies in the spectrum, such as a small departure from exact scale-invariance and a certain non-Gaussian character [3, 4], both of which can help reveal the underlying theory and dynamics of the fields during inflation. The observation of a small shift in the spectrum index $n-1 \neq 0$ is an obvious example [5] of scale invariance violation. Observations may also support a non-Gaussian character in the spectrum. Determining how these anomalies arise and how they relate to the inflation dynamics and character of the underlying theory, will depend on the model used. There are many models for inflation, in which the spectrum is generated (1) during inflation [6, 7, 8, 9, 10, (2) at the end of inflation [11, 12, 13], (3) after inflation by preheating [14, 15, 16] and reheating[17, 18] or (4) late after inflation by curvatons [19, 20, 21, 22] or by inhomogeneous phase transition[23].

For warm inflation, correction arises during evolution of the curvature perturbations that may be a significant source of these anomalies. We thus study the evolution of the curvature perturbations during warm inflation. In warm inflation, the effective potential of the inflaton fields depends on temperature $T$, which can be expressed as $V\left(\phi_{i}, T\right)$. The diffusion rate $\Gamma_{i}$ may also depend on $T$ and $\phi_{i}$. Therefore, the "trajectory" of warm inflation is given by both inflaton fields $\phi_{i}$ and $T$. The situation reminds us of multi-field inflation, in which the dynamics of a inflaton field is determined by the other inflaton fields, and vice versa. 2 The mutual dependency is important in the analysis of multi-field inflation. For warm inflation, the mutual dependency between inflaton fields and $T$ is

\footnotetext{
${ }^{2}$ See also Ref. 24] in which the evolution of the curvature perturbations is studied using numerical calculation.
} 
important. This paper considers warm inflation as an interesting application of multifield inflation. The evolution of linear perturbations about a FRW background metric has been discussed for supercooled inflation in Ref.[8], using only the local conservation of energy-momentum. The result does not depend on details of the inflation dynamics, thus it can be applied for warm inflation. In this paper, we apply the $\delta N$ formula [8] to warm inflation. The definitions of the $\delta N$ formula are discussed in the appendix of this paper.

We find that there is a significant correction to the curvature perturbation if the curvature perturbation at horizon crossing is expressed by the standard gaugeinvariant formula. We also find that a significant correction may arise from the perturbations of the radiation density.

Evolution of curvature perturbation in the $\delta N$ formalism

Evolution of the curvature perturbation can be studied without reference to specific inflationary dynamics or gravitational field equations [8]. In the following we demonstrate the calculation of the evolution of the curvature perturbations using the $\delta N$ formalism [6]. Calculations under this method are quite straightforward compared with calculations based on the time-derivative of the curvature perturbations on spatially flat hypersurfaces.

In standard (supercooled) inflation, the spectrum of the curvature perturbation $\mathcal{P}_{\mathcal{R}}(k)$ for the (adiabatic) inflaton field $\phi$ is given by

$$
\mathcal{P}_{\mathcal{R}}(k)=\left(\frac{H}{\dot{\phi}}\right)^{2}\left(\frac{H}{2 \pi}\right)^{2},
$$

where the right-hand side is evaluated at the epoch of the horizon exit $k=a H$. Here $H$ is the Hubble parameter and $a$ is the cosmic-scale factor. Here the comoving curvature perturbation $\mathcal{R}$ is considered, which is related to the curvature perturbation on uniformdensity hypersurfaces $\zeta$ as $\mathcal{R} \simeq-\zeta$ at large scales. The above equation is not exact in the warm inflation scenario, if the gauge-invariant combinations for the curvature perturbation are defined by

$$
\begin{aligned}
\zeta & =-\psi-H \frac{\delta \rho}{\dot{\rho}} \\
\mathcal{R} & =\psi-H \frac{\delta q}{\rho+p}
\end{aligned}
$$

where $\delta q=-\dot{\phi} \delta \phi$ is the momentum perturbation satisfying

$$
\epsilon_{m}=\delta \rho-3 H \delta q
$$


Here $\epsilon_{m}$ is the perturbation of the comoving density, satisfying the evolution equation

$$
\epsilon_{m}=-\frac{1}{4 \pi G} \frac{k^{2}}{a^{2}} \Psi,
$$

where $\Psi$ is related to the shear perturbation and assumed to be finite in this paper. The $\delta q=0$ hypersurfaces are identical to uniform density hypersurfaces $(\delta \rho=0)$ at large scales $\left(\epsilon_{m} \equiv \delta \rho-3 H \delta q \simeq 0\right)$. Linear scalar perturbations of a Friedman-RobertsonWalker(FRW) background are considered:

$$
d s^{2}=-(1+2 A) d t^{2}+2 a^{2}(t) \nabla_{i} B d x^{i} d t+a^{2}(t)\left[(1-2 \psi) \gamma_{i j}+2 \nabla_{i} \nabla_{j} E\right] d x^{i} d x^{j}
$$

Here $\rho$ and $p$ denote the energy density and the pressure during inflation. The comoving curvature perturbation on spatially flat hypersurfaces $\psi=0$ is expressed as

$$
\begin{aligned}
\mathcal{R} & =-H \frac{\delta q}{\rho+p} \\
& =-H \frac{\delta q}{\dot{\phi}^{2}+T s} \\
& \sim-H \frac{\delta q}{\dot{\phi}^{2}} \times\left(1+r_{\Gamma}\right)^{-1}
\end{aligned}
$$

where the differences from the standard supercooled inflation arise in the entropy $(s)$ and the damping term $(\Gamma)$, as will be defined and discussed in Sec.2. The difference between the one obtained in previous study of warm inflation will be explained in Sec.2 by considering the evolution of the curvature perturbations. In warm inflation, the inflation trajectory is determined by both $\phi$ and $T$, which leads to an interesting realization of "multi-field inflation". In fact, in supercooled inflation, multi-field model is considered using the total energy density. In this respect, it would be interesting to consider warm inflation as an interesting application of multi-field inflation.

The evolution of the curvature perturbation at large scales is found to be given by the non-adiabatic pressure perturbation $\delta p_{\text {nad }}$;

$$
\dot{\zeta} \simeq-H \frac{\delta p_{\text {nad }}}{\rho+p}
$$

where $\zeta$ and $\mathcal{R}$ coincide $(\zeta \simeq-\mathcal{R})$ at large scales and $\delta p_{\text {nad }} \equiv\left[\delta p-\frac{\dot{p}}{\dot{\rho}} \delta \rho\right]$ is a gaugeinvariant perturbation. This equation is valid independent of gravitational field equations. In fact, it can be found from Eq.(1.2) that

$$
\dot{\zeta}=-\dot{\psi}-\frac{d}{d t}\left[H \frac{\delta \rho}{\dot{\rho}}\right]
$$


Equations for the local conservation of energy momentum lead to the following useful expansion:

$$
\dot{\delta} \rho=-3 H(\delta \rho+\delta p)+(\rho+p)\left[3 \dot{\psi}-\nabla^{2}(\sigma+v+B)\right]
$$

where the scalar describing the shear is

$$
\sigma=\dot{E}-B
$$

and $\nabla^{i} v$ is the perturbed 3 -velocity of the fluid. Eq. (1.9) gives the equation for $\dot{\psi}$ in terms of the local conservation of energy momentum, which gives the simple equation for the evolution of $\zeta$ :

$$
\begin{aligned}
\dot{\zeta} & \simeq-\frac{\dot{\delta \rho}+3 H(\delta \rho+\delta p)}{3(\rho+p)}+\frac{d}{d t}\left[H \frac{\delta \rho}{3 H(\rho+p)}\right] \\
& =-\frac{H}{\rho+p}\left[\delta p-\frac{\dot{p}}{\dot{\rho}} \delta \rho\right] \\
& =-\frac{H}{\rho+p} \delta p_{\text {nad }}
\end{aligned}
$$

where $\nabla^{2}(\sigma+v+B)$ is neglected. To compare the above result with the $\delta N$ formalism[25], it is useful to define the perturbed expansion rate with respect to the coordinate time

$$
\delta \tilde{\theta} \equiv-3 \dot{\psi}+\nabla^{2} \sigma
$$

which can be used to define the $\delta N$ formalism. Choosing a gauge with a flat slicing at $t_{\text {ini }}$ and uniform density at $t$, and using $\zeta=-\psi$ for the specific choice of slice at $t$, the $\delta N$ formula is given by

$$
\zeta=\frac{1}{3} \int_{t_{\text {ini }}}^{t} \delta \tilde{\theta} d t=\delta N .
$$

The evolution equation for the $\delta N$ formalism is given by

$$
\begin{aligned}
\frac{d}{d t} \delta N & \equiv \frac{1}{3} \delta \tilde{\theta} \\
& \simeq-\dot{\psi} \\
& =\dot{\zeta}+\frac{d}{d t}\left(H \frac{\delta \rho}{\dot{\rho}}\right) \\
& =-\frac{H}{\rho+p} \delta p_{\text {nad }}+\frac{d}{d t}\left(H \frac{\delta \rho}{\dot{\rho}}\right)
\end{aligned}
$$

which shows that Eqs. (1.11) and (1.14) are consistent, as far as the relation is defined for the evolution of the curvature perturbation $\dot{\zeta}$ on a uniform density slice at $t$. The 
equation for the perturbed expansion rate $\delta \tilde{\theta}$ for $\delta N$ is practically valid for any gauge and slicing, but the $\delta N$ formula that explains the relation between $\zeta$ and $\delta N$ is defined for the specific choice of slice at $t$. The difference in the definition of the hypersurfaces will also lead to the difference in the boundary perturbation at the end of inflation, and the total $\delta N$ does not depend on the choice of hypersurfaces 3 In fact, the comoving density perturbation expressed in uniform density gauge shows that

$$
\epsilon_{m}=-3 H \delta q=-\frac{1}{4 \pi G} \frac{k^{2}}{a^{2}} \Psi
$$

which suggests that the perturbation of the adiabatic field $\delta \phi$ decays on uniform-density hypersurfaces. This result is consistent with the standard inflationary scenario in which $\phi_{e}$ that defines the the end of inflation is not perturbed. The decaying $\delta \phi$ is not important in supercooled inflation, while it causes significant evolution of the curvature perturbation in warm inflation, as will be discussed in Sec.2. In the standard inflationary scenario, $\delta N$ is created by the fluctuations of the inflaton field at the beginning of inflation. For the analysis of warm inflation in terms of the $\delta N$ formalism, it is useful to define $\dot{\zeta}_{N}$ using Eq.(1.9) as

$$
\begin{aligned}
\dot{\zeta}_{N} & \equiv \frac{d}{d t} \delta N \simeq-\dot{\psi} \\
& \simeq-H \frac{\delta(\rho+p)}{(\rho+p)}-H \frac{\dot{\delta} \rho}{3(\rho+p)} \\
& =-H \frac{\delta(\rho+p)}{(\rho+p)} \\
& =-H \frac{2 \delta K+\delta(T s)}{2 K+T s},
\end{aligned}
$$

where $T s$ appears for warm inflation but does not appear for supercooled inflation. This equation will be discussed in Sec.2.

As a result, in terms of the $\delta N$ formalism, the evolution of the curvature perturbation in warm inflation can be explained using the perturbations related to the inflaton kinetic term $(\delta K)$ and the radiation density $\delta(T s)$, where the radiation density is given by $\rho_{r}=$ $3 T s / 4$. In this paper, using the $\delta N$ formalism, a simple method for calculating the evolution of the curvature perturbation is considered for warm inflation.

\footnotetext{
${ }^{3}$ See Ref.[26] for an interesting example.
} 


\section{Evolution of the curvature perturbation}

\subsection{Warm inflation}

We first consider a homogeneous inflaton field interacting with thermal radiation[27]. Here we mainly follow the arguments in Ref.[27]. The thermodynamic potential is given by

$$
V(\phi, T)=-\frac{\pi^{2}}{90} g_{*} T^{4}+\frac{m(\phi, T) \phi^{2}}{2}+V_{0}(\phi)
$$

where $g_{*}$ is the effective number of thermal particles. The evolution equation for the inflaton field is given by

$$
\ddot{\phi}+(3 H+\Gamma) \dot{\phi}+V(\phi, T)_{\phi}=0
$$

where $\Gamma$ is the damping terms and $H$ is the expansion rate of the Universe. Here the subscript denotes the derivative with respect to the field. The strength of the thermal damping is measured by the rate $r$ given by

$$
r_{\Gamma} \equiv \frac{\Gamma}{3 H}
$$

which can be used to rewrite the field equation as

$$
\ddot{\phi}+3 H\left(1+r_{\Gamma}\right) \dot{\phi}+V(\phi, T)_{\phi}=0
$$

The typical situation for warm inflaton is defined by $r_{\Gamma} \gg 1$. The production of entropy during inflation is caused by the dissipation of the inflaton motion, where the entropy $s$ is defined by the thermodynamic equation;

$$
s \equiv-V(\phi, T)_{T}
$$

where the subscript denotes the derivative with respect to the temperature. From the energy-momentum tensor, it is found that the energy density $\rho$ and the pressure $p$ are given by

$$
\begin{aligned}
& \rho=K+V+T s \\
& p=K-V,
\end{aligned}
$$

where $K \equiv \frac{1}{2} \dot{\phi}^{2}$ is the contribution from the kinetic term of the inflaton field, and the Friedman equation follows

$$
H^{2}=\frac{1}{3 M_{p}^{2}}(K+V+T s)
$$


The slow-roll approximation is very useful in estimating the order of magnitude of the physical quantities:

$$
\begin{aligned}
\dot{\phi} & \simeq-\frac{V_{\phi}}{3 H\left(1+r_{\Gamma}\right)} \\
T s & \simeq 2 r_{\Gamma} K \\
\rho+P & =2 K+T s \simeq 2\left(1+r_{\Gamma}\right) K
\end{aligned}
$$

where the second equation is obtained from the evolution equation for the radiation energy density. On spatially flat hypersurfaces $\psi=0$, the comoving curvature perturbation created during warm inflaton is expressed as

$$
\begin{aligned}
\mathcal{R} & =-H \frac{\delta q}{\rho+p} \\
& =-H \frac{\delta q}{\dot{\phi}^{2}+T s} \\
& \sim-H \frac{\delta q}{\dot{\phi}^{2}} \times\left(1+r_{\Gamma}\right)^{-1}
\end{aligned}
$$

The important source of density perturbations in warm inflation is thermal fluctuations. A comoving mode of thermal fluctuations during warm inflation is created by thermal noise. The behavior of a scalar field interacting with radiation can be studied by using the Langevin equation

$$
-\nabla \phi(x, t)+\Gamma \dot{\phi}(x, t)+V_{\phi}=\xi(x, t)
$$

where $\xi$ denotes a source of stochastic noise. From the Langevin equation, the root-mean square fluctuation amplitude of the inflaton field $\delta \phi$ after the freeze out at $k / a \sim(\Gamma H)^{1 / 2}$ is obtained to be

$$
\delta \phi \sim-\left(\frac{\pi}{4}\right)(\Gamma H)^{1 / 4} T^{1 / 2} \sim r_{\Gamma}^{1 / 4} r_{T}^{1 / 2} H,
$$

where $r_{T}$ denotes the ratio between $T$ and $H$, defined by $r_{T} \equiv T / H$. Thermal fluctuation of the radiation density is also important in warm inflation. It has been calculated to be 27]

$$
\delta \rho_{r} \sim g_{*}^{1 / 2}\left(\frac{k}{a}\right)^{3 / 2} T^{5 / 2} .
$$

In warm inflation with single inflaton field, the total energy density and pressure 
perturbations for the first-order approximation are given by [27]

$$
\begin{aligned}
\delta \rho & \simeq \delta K+V_{\phi} \delta \phi+T \delta s \\
\delta p & \simeq \delta K-V_{\phi} \delta \phi+s \delta T .
\end{aligned}
$$

For two-field inflation with $\left(\phi_{1}, \phi_{2}\right)$, one may redefine the adiabatic inflaton field $\phi_{\sigma}$ and the entropy field $\phi_{s}$. Then $V\left(\phi_{\sigma}, \phi_{s}, T\right) \simeq V_{\phi_{\sigma}} \delta \phi_{\sigma}+V_{\phi_{s}} \delta \phi_{s}+V_{T} \delta T$ is obtained, which leads to

$$
\begin{aligned}
& \delta \rho \simeq \delta K+V_{\phi_{\sigma}} \delta \phi_{\sigma}+V_{\phi_{s}} \delta \phi_{s}+T \delta s \\
& \delta p \simeq \delta K-V_{\phi_{\sigma}} \delta \phi-V_{\phi_{s}} \delta \phi_{s}+s \delta T
\end{aligned}
$$

where the entropy field satisfies $\dot{\phi}_{s}=0$.

Assuming that $\phi$ is identified with the adiabatic inflaton field, the momentum perturbation is given by

$$
\delta q=-\dot{\phi} \delta \phi
$$

Considering the backreaction from radiation, the velocity perturbation is given by [1]

$$
\delta \dot{\phi} \sim \frac{k^{2}}{a^{2} H^{2}} H^{2} r_{\Gamma}^{-3 / 4} r_{T}^{1 / 2}
$$

This may lead to second-order perturbation $\delta^{(2)} q \simeq H^{2} r_{\Gamma}^{-3 / 4} r_{T}^{1 / 2} \delta \phi \times k^{2} /(a H)^{2}$. The second-order perturbation $\delta q^{(2)}$ may also appear for fields that are not inflaton. The second-order perturbation is disregarded in this paper for simplicity, but it may become significant when multiplied by a huge number of the fields in the model. Note also that the momentum perturbation proportional to $k^{2} / a^{2}$ vanishes at large scales. The comoving density perturbation is defined by a gauge-invariant quantity

$$
\begin{aligned}
\epsilon_{m} & \equiv \delta \rho-3 H \delta q \\
& =\delta K+\left(\delta V+\delta[T s]-\left(1+r_{\Gamma}\right)^{-1} V_{\phi} \delta \phi\right)-\left(1+r_{\Gamma}\right)^{-1} \ddot{\phi} \delta \phi,
\end{aligned}
$$

where the last equation is obtained using the field equation. The comoving density perturbation satisfies the evolution equation

$$
\epsilon_{m}=-\frac{1}{4 \pi G} \frac{k^{2}}{a^{2}} \Psi
$$


where $\Psi$ is related to the shear perturbation. Here we assume $\Psi$ reaches a finite value at large scales, which leads to $\epsilon_{m} \simeq 0$. To estimate $\dot{\zeta}_{N}$, the perturbation $\delta K$ needs to be obtained from the above equations. It is given by

$$
\delta K \simeq-\delta V-\delta[T s]+\left(1+r_{\Gamma}\right)^{-1} V_{\phi} \delta \phi
$$

where $\ddot{\phi}$ has been disregarded. The perturbation $\delta K$ in the $\delta N$ formula, which is caused by perturbations in the kinetic term, can thus be replaced using the equation for the comoving energy density perturbation. It follows that

$$
\begin{aligned}
-\dot{\psi} & \simeq H \frac{2\left\{\delta V+\delta[T s]-\left(1+r_{\Gamma}\right)^{-1} V_{\phi} \delta \phi\right\}-\delta(T s)}{2\left(1+r_{\Gamma}\right) K} \\
& \simeq-H \frac{3 s \delta T+T \delta s}{2\left(1+r_{\Gamma}\right) K}+2 H \frac{V_{\phi_{s}} \delta \phi_{s}}{\left(1+r_{\Gamma}\right) K}+H \frac{r_{\Gamma} V_{\phi}}{\left(1+r_{\Gamma}\right)^{2} K} \delta \phi
\end{aligned}
$$

The second term is important when there is a bend in the inflation trajectory 4 In the third term $\delta \phi$ is defined on uniform-density hypersurfaces. Considering two different definitions of $\delta t$, which are motivated by the $\delta N$ formula defined for different hypersurfaces;

$$
\begin{aligned}
\delta t_{\delta \phi=0} & \equiv \frac{\delta \phi}{\dot{\phi}} \\
\delta t_{\delta \rho=0} & \equiv \frac{\delta \rho}{\dot{\rho}} \simeq-\frac{\delta q}{\rho+p} \simeq \frac{\delta \phi}{\left(1+r_{\Gamma}\right) \dot{\phi}}
\end{aligned}
$$

we find that there is a significant discrepancy between these two definitions. Namely, for strongly dissipating warm inflation (SDWI, $r_{\Gamma} \gg 1$ ), we find $\delta t_{\delta \phi=0} \gg \delta t_{\delta \rho=0}$ at large scales where $\epsilon_{m} \simeq 0$. This discrepancy cannot appear in supercooled inflation, in which the diffusion rate vanishes by definition. As a result, in supercooled inflation, there is no significant evolution caused by $\delta \phi$, but in strongly dissipating warm inflation, $\delta \phi$ in the third term is still as large as Eq. (2.13) and raises significant contribution.

Using the above equations and the $\delta N$ formula, it is possible to examine the order of magnitude of $\dot{\zeta}_{N}$ that can be caused by perturbations of the radiation and the third term.

\footnotetext{
${ }^{4}$ We considered two-field warm inflation to show what happens at the bend of the trajectory in warm inflation.
} 
They can be estimated as

$$
\begin{aligned}
\dot{\zeta}_{N}^{(r a d)} & \sim H \frac{\delta \rho_{r}}{\left(1+r_{\Gamma}\right) K} \\
& \sim H\left(\frac{k}{a H}\right)^{3 / 2} \frac{T^{5 / 2} H^{3 / 2}}{T^{4}} \frac{r_{\Gamma}}{1+r_{\Gamma}} \\
& \sim H\left(\frac{k}{a H}\right)^{3 / 2} \frac{r_{\Gamma}}{r_{T}^{3 / 2}\left(1+r_{\Gamma}\right)}
\end{aligned}
$$

and

$$
\begin{aligned}
\dot{\zeta}_{N}^{3 r d} & \sim H^{2} \frac{r_{\Gamma} V_{\phi} \delta \phi}{\left(1+r_{\Gamma}\right)^{2} K} \\
& \sim H^{2} \frac{r_{\Gamma}}{1+r_{\Gamma}} \frac{\delta \phi}{\dot{\phi}}
\end{aligned}
$$

Although the correction arising from $\delta \rho_{r}$ decays at large scales as $\delta \rho_{r} \propto(k / a)^{3 / 2}$, it may cause significant correction during warm inflation. Namely, a decaying effect expressed by $\dot{\zeta}_{N} \propto H \delta C e^{-A H t}$ can leave a significant effect after a time-integral;

$$
\Delta \zeta_{N}\left(t_{*}\right) \sim \int_{0}^{t_{*}} H \delta C e^{-A H t} d t \sim \frac{C}{A}\left(1-e^{-A H t_{*}}\right)
$$

Then, due to the constancy of the curvature perturbations at large scales, the correction $\Delta \zeta_{N}$ remains at large scales. Applying the above result to Eq.(2.25), $\Delta \zeta_{N}^{(\mathrm{rad})}$ at large scales is found to be given by

$$
\Delta \zeta_{N}^{(r a d)} \sim \int_{0}^{t} \dot{\zeta}_{N}^{(r a d)} d t \sim \frac{r_{\Gamma}}{r_{T}^{3 / 2}\left(1+r_{\Gamma}\right)}
$$

where $k=a H$ at $t=0$. We also find for the third term;

$$
\Delta \zeta_{N}^{(3 r d)} \sim \int_{0}^{t} \dot{\zeta}_{N}^{(3 r d)} d t \sim \frac{r_{\Gamma}^{7 / 4}}{r_{T}^{3 / 2}\left(1+r_{\Gamma}\right)}
$$

$\Delta \zeta_{N}^{(3 r d)}$ dominates the curvature perturbations for $r_{\Gamma} \gg 1$, but these two terms are comparable when $r_{\Gamma} \simeq 1$, near the boundary between the weakly dissipating $\left(r_{\Gamma} \ll 1\right)$ and strongly dissipating $\left(r_{\Gamma} \gg 1\right)$ phases of warm inflation.

Considering the $\mathrm{CMB}$ normalization $\mathcal{P}_{\mathcal{R}}{ }^{1 / 2} \sim 10^{-5}$, it is straightforward to find that $\Delta \zeta_{N}$ may dominate the curvature perturbation for realistic parameter space for the warm inflation scenario. To avoid the excessive creation of the curvature perturbation in terms of the evolution after the horizon exit, the condition $r_{T}^{3 / 2}\left(1+r_{\Gamma}\right) r_{\Gamma}^{-7 / 4}>10^{5}$ must be 
imposed for warm inflation. For large $r_{\Gamma}$ and $r_{T}$, the result can be simplified to give the condition $r_{T}^{3 / 2} r_{\Gamma}^{-3 / 4}>10^{5}$, which is required to satisfy $\Delta \zeta_{N}<10^{-5}$ but leads to a significant reduction of the conventional curvature perturbations created at the horizon exit.

There are two important consequences in the analysis. One is that the conventional curvature perturbation defined by $\mathcal{R} \sim H \delta q /(\rho+P)$ for spatially flat hypersurfaces evolves during inflation to give significant correction $\Delta \zeta^{(3 r d)} \sim H \delta \phi / \dot{\phi}$, which finally meet the previous study of warm inflation. The gauge invariance of the curvature perturbations and the evolution is now clear in this formalism. 5 The other is that evolution during inflation may create another significant correction $\Delta \zeta_{N}^{(r a d)}$, which has been overlooked in previous study but may be as large as $\Delta \zeta_{N}^{(r a d)} \simeq \Delta \zeta^{(3 r d)}$ for $r_{\Gamma} \simeq 1$. This term may leave significant signature in cosmological perturbations when warm inflation passes from strongly dissipating phase to weakly dissipating phase, which may help reveal the mechanism of inflation.

$\Delta \zeta_{N}$ as the source of the cosmological perturbations

Considering Eq. (2.11) and Eq.(2.13), we find for the curvature perturbation created at the horizon exit;

$$
\begin{aligned}
\mathcal{R}_{i n i} & \sim \frac{H \delta \phi}{\sqrt{\dot{\phi}^{2}}\left(1+r_{\Gamma}\right)} \\
& \sim \frac{H^{2}}{T^{2}} \frac{r_{\Gamma}^{3 / 4} t_{T}^{1 / 2}}{\left(1+r_{\Gamma}\right)} \\
& \sim \frac{r_{\Gamma}^{3 / 4}}{\left(1+r_{\Gamma}\right) r_{T}^{3 / 2}} \sim r_{\Gamma}^{-1 / 4} r_{T}^{-3 / 2}
\end{aligned}
$$

If the CMB normalization is applied to $\Delta \zeta_{N}\left(\right.$ not to $\left.\mathcal{R}_{\text {ini }}\right)$, it is found that

$$
\frac{r_{\Gamma}^{7 / 4}}{r_{T}^{3 / 2}\left(1+r_{\Gamma}\right)} \sim 10^{-5},
$$

where $\mathcal{R}_{i n i} \sim r_{\Gamma}^{-1 / 4} r_{T}^{-3 / 2}<10^{-5}$ is assumed for the domination by $\Delta \zeta_{N}$. The condition for the successful creation of the curvature perturbation in terms of $\Delta \zeta_{N}$ is thus given by

$$
r_{T}^{-3 / 2} r_{\Gamma}^{7 / 4} \sim 10^{-5}
$$

\footnotetext{
${ }^{5}$ Note that the $\delta N$ perturbation defined by $H \delta \phi / \dot{\phi}$ is obviously different from the conventional gaugeinvariant definition of the curvature perturbation. See also the appendix of this paper.
} 
$\underline{\text { Inhomogeneous diffusion rate }}$

It would be useful to discuss another source for creating inhomogeneities of the expansion rate during warm inflation. Here we briefly show that inhomogeneities of the diffusion rate $\Gamma$, which may be induced by light (moduli) fields, may cause inhomogeneities of the expansion rate during warm inflation. In fact, if dissipation occurs into light degrees of freedom and it quickly thermalize into radiation, it is found that

$$
\dot{\rho}_{r}+4 H \rho_{r}=\Gamma \dot{\phi}^{2}
$$

and if the continuous dissipation keeps $\rho_{r}$ constant during warm inflation, it leads to

$$
4 \rho_{r} \simeq 3 r_{\Gamma} \dot{\phi}^{2}
$$

From the equation of motion, the slow-roll velocity of the inflaton field is found to be given by

$$
\dot{\phi} \simeq-\frac{V_{\phi}}{3 H\left(1+r_{\Gamma}\right)} .
$$

For $r_{\Gamma}>1$, the fluctuation of the slow-roll velocity that arises from the inhomogeneous diffusion rate is thus given by

$$
\frac{\delta \dot{\phi}}{\dot{\phi}} \simeq \frac{\delta \Gamma}{\Gamma}
$$

where the diffusion rate $\Gamma$ is diffent in diffent Hubble patches. The inhomogeneities of $\Gamma$ may also create $\delta \rho_{r}$ in terms of the dissipation given in Eq.(2.34). The inhomogeneous diffusion rate thus leads to inhomogeneities of the expansion rate, which creates curvature perturbations given by

$$
\Delta_{(\delta \Gamma)} \zeta_{N} \sim \frac{\delta \Gamma}{\Gamma}
$$

where the magnitude of the perturbations is determined by the model. A possible source of $\delta \Gamma$ is the perturbations related to entropy (moduli) fields. The diffusion rate may also depend on the temperature. The temperature perturbation decays after horizon crossing, however it may leave significant correction after time integration.

Introducing a light field $\sigma$, a specific example of the diffusion rate can be given by [28]

$$
\Gamma \sim C(\sigma) \frac{T^{3}}{\phi^{2}}
$$


which causes inhomogeneities of $\Gamma$ given by

$$
\begin{aligned}
\frac{\delta \Gamma}{\Gamma} & \sim \frac{C_{\sigma}}{C} \delta \sigma+3 \frac{\delta T}{T}-2 \frac{\delta \phi}{\phi} \\
& \sim \frac{C_{\sigma}}{C} \delta \sigma+3 r_{T}^{-3 / 2}-2 r_{\Gamma}^{1 / 4} r_{T}^{1 / 2} \frac{H}{\phi} .
\end{aligned}
$$

If is also possible to find significant non-gaussianity from $\delta \Gamma$, expanding the moduli-

dependent part as $\delta C \simeq C_{0}+C_{2} \frac{\sigma^{2}}{M_{*}^{2}}$, for example. It is useful to specify the level of nonGaussianity by the non-linear parameter $f_{N L}$, which is usually defined by the Bardeen potential $\Phi$,

$$
\Phi=\Phi_{\text {Gaussian }}+f_{N L} \Phi_{\text {Gaussian }}^{2}
$$

Using the Bardeen potential, the curvature perturbation $\zeta$ is given by

$$
\Phi=\frac{3}{5} \zeta
$$

Considering the expansion of $\delta N$ as

$$
\zeta \simeq N_{\phi} \delta \phi+N_{\sigma} \delta \sigma+\frac{1}{2} N_{\phi \phi} \delta \phi^{2}+\frac{1}{2} N_{\sigma \sigma} \delta \sigma^{2}+\ldots
$$

A useful simplification is 29

$$
f_{N L} \simeq\left(\frac{1}{1300} \frac{N_{\sigma \sigma}(\delta \sigma)^{2}}{N_{\phi}^{2}(\delta \phi)^{2}}\right)^{3}
$$

The non-linear parameter is estimated as

$$
f_{N L} \sim 10^{21} C_{2}^{3}\left(\frac{\delta \sigma}{M_{*}}\right)^{6},
$$

which may be as large as $f_{N L} \sim 10$.

\subsection{Decay into non-relativistic matter}

If the non-relativistic matter $\chi$ created by the dissipation of the inflaton motion does not decay, the evolution equation for the inflaton is given by

$$
\ddot{\phi}+(3 H+\Gamma) \dot{\phi}+V_{\phi}^{e f f}=0
$$

Introducing entropy field $\mathcal{M}$ (moduli) in addition to the adiabatic inflaton field $\phi$, the effective potential can be expressed as

$$
\begin{aligned}
V^{e f f} & \equiv V_{0}(\phi)+\rho_{\chi} \\
& \simeq V_{0}(\phi)+M_{\chi}(\phi, \mathcal{M}) n_{\chi}
\end{aligned}
$$


Here $M_{\chi}$ and $n_{\chi}$ are the effective mass and the number density of the non-relativistic matter $\chi$. If the continuous dissipation keeps the energy density $\rho_{\chi}$ constant for a time period during inflation, and also the pressure is given by using a constant $\omega$ as $p_{\chi}=\omega \rho_{\chi}$, the energy density and the pressure are given by

$$
\begin{aligned}
& \rho=K+V^{e f f} \\
& p=K-V^{e f f}+(1+\omega) \rho_{\chi} .
\end{aligned}
$$

Again, slow-roll approximation is useful in estimating the order of magnitude of the physical quantities:

$$
\begin{aligned}
\dot{\phi} & \simeq-\frac{V_{\phi}}{3 H\left(1+r_{\Gamma}\right)} \\
(1+\omega) \rho_{\chi} & \simeq 2 r_{\Gamma} K \\
\rho+P & =2 K+(1+\omega) \rho_{\chi} \simeq 2\left(1+r_{\Gamma}\right) K .
\end{aligned}
$$

Therefore, on spatially flat hypersurfaces $\psi=0$, comoving curvature perturbation created during warm inflaton is expressed as

$$
\begin{aligned}
\mathcal{R} & =-H \frac{\delta q}{\rho+p} \\
& \sim-H \frac{\delta q}{\dot{\phi}^{2}} \times\left(1+r_{\Gamma}\right)^{-1} .
\end{aligned}
$$

Unlike warm inflaton, the source of the cosmological perturbations is not thermal fluctuations but the conventional field perturbations created during inflation. The comoving density perturbation is given by

$$
\begin{aligned}
\epsilon_{m} & \equiv \delta \rho-3 H \delta q \\
& =\delta K+\left(\delta V^{e f f}-r_{\Gamma}^{-1} V_{\phi}^{e f f} \delta \phi\right)-r_{\Gamma}^{-1} \ddot{\phi} \delta \phi
\end{aligned}
$$

It follows that

$$
\begin{aligned}
-\dot{\psi} & \simeq H \frac{2\left(\delta V^{e f f}-r_{\Gamma}^{-1} V_{\phi} \delta \phi\right)-(1+\omega) \delta \rho_{\chi}}{2\left(1+r_{\Gamma}\right) K} \\
& \sim H \frac{(1-\omega) \delta \rho_{\chi}}{2\left(1+r_{\Gamma}\right) K} .
\end{aligned}
$$

Using the above equations and the $\delta N$ formula, the evolution of the curvature perturbation caused by the inhomogeneities of the non-relativistic matter $\chi$ is given by

$$
\dot{\zeta}_{N} \sim H \frac{(1-\omega)}{(1+\omega)} \frac{r_{\Gamma}}{1+r_{\Gamma}} \frac{\delta \rho_{\chi}}{\rho_{\chi}} .
$$

Here $\delta \rho_{\chi}$ is sourced by $\delta \Gamma$, which arises due to the inhomogeneities of the entropy fields. 


\section{Conclusions and discussions}

In warm inflation with single-field inflaton, the effective potential of the inflaton field depends on the temperature $T$, which can be expressed as $V(\phi, T)$. The dissipation rate $\Gamma$ may also depend on $T$ and $\phi$. In this sense, the "trajectory" of inflation is given by both $\phi$ and $T$.

The situation reminds us of multi-field inflation, in which the dynamics of a inflaton field is determined by the dynamics of the other inflaton fields, and vice versa. To compare the situations, first consider two-field inflation with a rapid-rolling inflaton $\phi_{F}$ that reaches its minimum during inflation and a slow-rolling inflaton $\phi$ that determines the number of e-foldings of the inflationary expansion. Namely, on spatially flat hypersurfaces, the conventional definition of the curvature perturbation is given by

$$
\mathcal{R}_{\text {multi }}^{(i n i)}=-H \frac{\delta q}{\rho+P}=H \frac{\dot{\phi}_{F} \delta \phi_{F}+\dot{\phi} \delta \phi}{\dot{\phi}_{F}^{2}+\dot{\phi}^{2}} \simeq H \frac{\dot{\phi} \delta \phi}{\dot{\phi}_{F}^{2}}
$$

where $\delta \phi_{F} \simeq 0$ on the steep potential. In this case the evolution during inflation is crucial, since at the "bend" of the inflation trajectory it gives

$$
\Delta \mathcal{R}_{m u l t i} \simeq H \frac{\delta \phi}{\dot{\phi}} \gg \mathcal{R}_{m u l t i}^{(i n i)}
$$

which is consistent with the $\delta N$ formalism based on $\delta \phi$ and $\dot{\phi}$. A similar situation appears in warm inflation, in which the curvature perturbation is given by

$$
\mathcal{R}_{\text {warm }}^{(i n i)}=-H \frac{\delta q}{\rho+P}=H \frac{\dot{\phi} \delta \phi}{\dot{\phi}^{2}+T s} \simeq H \frac{\dot{\phi} \delta \phi}{T s},
$$

where the thermal perturbations disappear at large scales. Again, the evolution during inflation is crucial, since from a decaying component of warm inflation we find

$$
\Delta \mathcal{R}_{w a r m} \simeq H \frac{\delta \phi}{\dot{\phi}} \gg \mathcal{R}_{w a r m}^{(i n i)}
$$

which is consistent with the previous study based on $\delta \phi$ and $\dot{\phi}$.

We also found that a significant correction may arise from the perturbations of the radiation density, which raises significant correction at the intermediate region between strongly dissipating warm inflation (SDWI) and weakly dissipating warm infla$\operatorname{tion}(\mathrm{WDWI})$. 
This paper considers warm inflation as an interesting application of multi-field inflation. The $\delta N$ perturbation defined for a $\phi$-constant hypersurface may give the required curvature perturbation. However, considering the conventional definition of the curvature perturbation given in Eq. (1.2), which is manifestly gauge-invariant, there is a serious discrepancy between these two definitions. The problem must be solved explicitly by considering gauge-invariant evolution of the curvature perturbation, which is defined by Eq. (1.2). Since a $\phi$-constant hypersurface is identical to uniform density hypersurface at large scales, the evolution at small scales must be very important in solving this problem. Consideration of the decaying term in the evolution of the curvature perturbation is a new idea, which is necessary in solving this problem. A solution to this problem is one of the main result obtained in this paper.

Significant corrections to the curvature perturbation are raised from the small-scale perturbations of the radiation and from the decaying $\delta \phi$, which are found to dominate the curvature perturbation. The one from the decaying $\delta \phi$ reproduces the $\delta N$ perturbation for $\phi$-constant hypersurfaces, and the other from the radiation creates a new contribution, which can nearly dominate the curvature perturbation for $r_{\Gamma} \simeq 1$.

It is also shown that significant non-gaussianity may be created from the inhomogeneities of the dissipation rate, which appears independent of the conventional curvature perturbations.

The short-scale corrections that we have considered in this paper may be dubbed the non-equilibrium corrections. Other kinds of corrections have already been discussed for supercooled inflation in Ref.[6], and these may be significant in string cosmological models.

\section{Acknowledgment}

We wish to thank K.Shima for encouragement, and our colleagues at Tokyo University for their kind hospitality. 


\section{A "Local" and "global" definitions of the $\delta N$ formula}

In this paper we considered the $\delta N$ formula defined for the uniform density hypersurfaces at a time-slice and calculated the evolution of $\delta N$. In this appendix, the $\delta N$ formula defined in this way is denoted by $\delta N_{\text {local }}$. It is also possible to define $\delta N$ using the number of e-foldings $N_{e}$, which is defined using the interval between the horizon exit at $t=t_{\text {ini }}$ and the end of inflation at $t=t_{e}$. We denote the latter definition by $\delta N_{\text {global }}$. Obviously, it is impossible to calculate the "evolution" (not the scale-dependence) of $\delta N_{\text {global }} 6$

$\delta N_{\text {local }}$ is defined so that it is related to the curvature perturbations at the time-slice. If one wants to understand the evolution of the curvature perturbations in terms of the $\delta N$ formalism, one should consider $\delta N_{\text {local }}$ for the definition of the $\delta N$ formula.

One may find a discrepancy between $\delta N_{\text {local }}$ and $\delta N_{\text {global }}$, which must be solved by considering the evolution of $\delta N_{\text {local }}$. A simple example of the inflaton trajectory in typical multi-field inflation is shown in Figure 1. Here we consider two-field inflation with a separable potential. The $\phi_{2}$-potential is steep and it rolls much faster than $\phi_{1}$. Following the standard definition of the curvature perturbations, we find that the curvature perturbations at a time-slice is given by

$$
\mathcal{R}_{i n i}=-H \frac{\delta q}{\rho+P}=H \frac{\dot{\phi}_{1} \delta \phi_{1}+\dot{\phi}_{2} \delta \phi_{2}}{\dot{\phi}_{1}^{2}+\dot{\phi}_{2}^{2}}=H \frac{\delta \phi_{\sigma}}{\dot{\phi}_{\sigma}},
$$

where the adiabatic inflaton is defined by $\dot{\phi}_{\sigma}^{2} \equiv \dot{\phi}_{1}^{2}+\dot{\phi}_{2}^{2}$. However, one may find from the trajectory (See figure 1) that the number of e-foldings can be determined exclusively by $\phi_{1}$, and there may not be a $\delta \phi_{2}$-dependence in $\delta N_{\text {global }} 7$ On the other hand, if one defines the curvature perturbations at a time slice by $\mathcal{R} \sim H \delta \phi_{1} / \dot{\phi}_{1}$, one immediately finds that the definition is not manifestly gauge-invariant. In this case the evolution during inflation is crucial, since at a "bend" of the inflation trajectory the evolution is given by

$$
\Delta \mathcal{R} \simeq H \frac{\delta \phi_{1}}{\dot{\phi}_{1}} \gg \mathcal{R}_{i n i}
$$

One might claim that the argument related to the evolution at the "bend" of the trajectory gives a trivial argument for the curvature perturbations, claiming that it gives a trivial

\footnotetext{
${ }^{6}$ Note also that the curvature perturbations defined at the horizon exit $\left(\mathcal{R}_{\text {ini }}\right)$ has the trivial $k$ dependence, which must be distinguished from the "evolution" defined in this paper.

${ }^{7}$ More examples can be found in the first paper in [13], in which the fluctuations at the end-boundary are also taken into account for the calculation of $\delta N_{\text {global }}$.
} 
result in view of $\delta N_{\text {golbal }}$. However, it is not obvious if the intuitive argument based on $\delta N_{\text {global }}$ always gives the correct result. Therefore, the source that explains the gap in these definitions must be identified from the calculation of the evolution of $\delta N_{\text {local }}$.

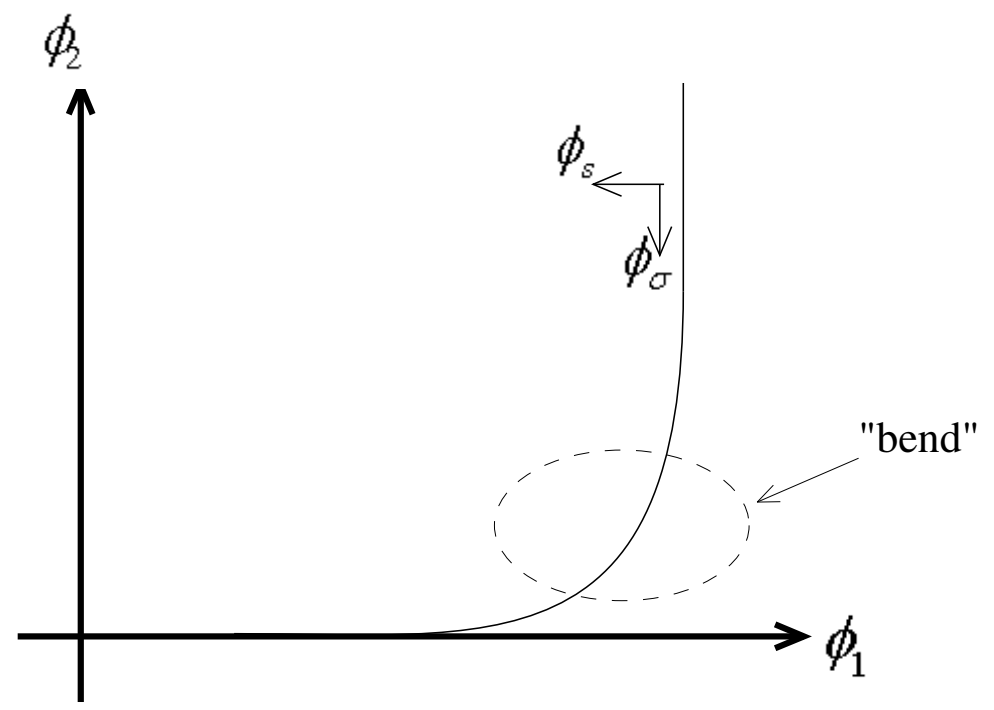

Figure 1: The curved line represents a trajectory in two-field inflation. $\phi_{2}$ rolls faster than $\phi_{1}$ and is significant at the beginning. However, soon $\phi_{2}$ reaches its minimum and the dynamics of $\phi_{2}$ is negligible near the end of inflation. Note also that it is possible to consider a $\phi_{2}$ field that is heavy and oscillating during inflation. In this case, $\phi_{2}$ generates "many bends" in the trajectory. See also the first paper in Ref.[13] for more details of the global calculation of $\delta N$.

Applying the same argument to warm inflation, it is easy to find that the usual definition of the curvature perturbations in warm inflationary models are based on $\delta N_{\text {global }}$, while in this paper we calculated the evolution of $\delta N_{\text {local }}$ to find that the calculation based on the intuitive argument of $\delta N_{\text {global }}$ is consistent with the explicitly gauge-invariant definition of the curvature perturbations at a time-slice. In our calculation we also introduced another inflaton field to show what happens at the bend of the trajectory in warm inflation. Note that we are not considering a counter-example that may ruin the usual argument based on $\delta N_{\text {global }}$.

The end-boundary of warm inflation is usually defined by the field value at a critical point where slow-roll condition is violated. However, more generically the end-boundary can be defined by a critical temperature, as will be discussed in our forthcoming paper [30]. 


\section{References}

[1] A. Berera, "Warm Inflation," Phys. Rev. Lett. 75, 3218 (1995) arXiv:astro-ph/9509049. A. Berera, "The warm inflationary universe," Contemp. Phys. 47, 33 (2006) [arXiv:0809.4198 [hep-ph]]; M. Bellini, "Fresh inflation with increasing cosmological parameter," Phys. Rev. D 67, 027303 (2003) arXiv:gr-qc/0211044]; M. Bellini, "Primordial fluctuations of the metric in the warm inflation scenario," Nuovo Cim. B 115, 369 (2000) arXiv:gr-qc/0005072.

[2] A. Hosoya and M. a. Sakagami, "Time Development Of Higgs Field At Finite Temperature," Phys. Rev. D 29, 2228 (1984).

[3] N. Bartolo, E. Komatsu, S. Matarrese and A. Riotto, "Non-Gaussianity from inflation: Theory and observations," Phys. Rept. 402, 103 (2004) arXiv:astro-ph/0406398.

[4] A. P. S. Yadav and B. D. Wandelt, "Evidence of Primordial Non-Gaussianity $\left(f_{\mathrm{NL}}\right)$

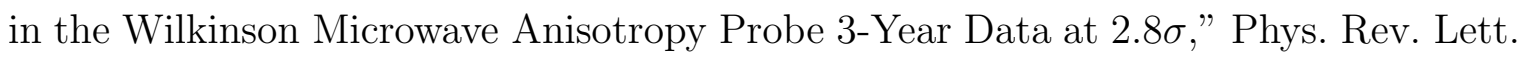
100, 181301 (2008) arXiv:0712.1148.

[5] A. R. Liddle and D. H. Lyth, "Cosmological inflation and large-scale structure," Cambridge, UK: Univ. Pr. (2000) 400 p.

[6] T. Matsuda, "Delta-N formalism for the evolution of the curvature perturbations in generalized multi-field inflation," arXiv:0906.2525 [hep-th]; T. Matsuda, "Modulated Inflation," Phys. Lett. B 665, 338 (2008) arXiv:0801.2648 [hep-ph]]; T. Matsuda, "Modulated inflation from kinetic term," JCAP 0805, 022 (2008) arXiv:0804.3268 [hep-th]]; T. Matsuda, "Running spectral index from shooting-star moduli," JHEP 0802, 099 (2008) [arXiv:0802.3573 [hep-th]]; T. Matsuda, "Successful D-term inflation with moduli," Phys. Lett. B 423, 35 (1998) arXiv:hep-ph/9705448.

[7] L. Kofman, "Probing string theory with modulated cosmological fluctuations," arXiv:astro-ph/0303614.

[8] D. Wands, K. A. Malik, D. H. Lyth and A. R. Liddle, "A new approach to the evolution of cosmological perturbations on largescales," Phys. Rev. D 62, 043527 
(2000) arXiv:astro-ph/0003278; D. H. Lyth and D. Wands, "Conserved cosmological perturbations," Phys. Rev. D 68, 103515 (2003) arXiv:astro-ph/0306498]; C. Gordon, D. Wands, B. A. Bassett and R. Maartens, "Adiabatic and entropy perturbations from inflation," Phys. Rev. D 63, 023506 (2001) [arXiv:astro-ph/0009131].

[9] T. Matsuda, "Curvatons and inhomogeneous scenarios with deviation from slow-roll," JCAP 0812 (2008) 001 arXiv:0811.1318 [hep-ph]].

[10] A. C. Vincent and J. M. Cline, "Curvature Spectra and Nongaussianities in the Roulette Inflation Model," JHEP 0810, 093 (2008) [arXiv:0809.2982 [astro-ph]]. J. R. Bond, L. Kofman, S. Prokushkin and P. M. Vaudrevange, "Roulette inflation with Kaehler moduli and their axions," Phys. Rev. D 75, 123511 (2007) arXiv:hep-th/0612197.

[11] F. Bernardeau, L. Kofman and J. P. Uzan, "Modulated fluctuations from hybrid inflation," Phys. Rev. D 70, 083004 (2004) arXiv:astro-ph/0403315.

[12] D. H. Lyth, "Generating the curvature perturbation at the end of inflation," JCAP 0511, 006 (2005) arXiv:astro-ph/0510443.

[13] T. Matsuda, "Elliptic inflation: Generating the curvature perturbation without slowroll," JCAP 0609, 003 (2006) arXiv:hep-ph/0606137]; D. H. Lyth and A. Riotto, "Generating the curvature perturbation at the end of inflation in string theory," Phys. Rev. Lett. 97, 121301 (2006) arXiv:astro-ph/0607326]; T. Matsuda, "Non-tachyonic brane inflation," Phys. Rev. D 67, 083519 (2003) arXiv:hep-ph/0302035.

[14] E. W. Kolb, A. Riotto and A. Vallinotto, "Curvature perturbations from broken symmetries," Phys. Rev. D 71, 043513 (2005) arXiv:astro-ph/0410546]; T. Matsuda, "Generating the curvature perturbation with instant preheating," JCAP 0703, 003 (2007) arXiv:hep-th/0610232; T. Matsuda, "Generating curvature perturbations with MSSM flat directions," JCAP 0706, 029 (2007) arXiv:hep-ph/0701024.

[15] T. Matsuda, "Non-standard kinetic term as a natural source of non-Gaussianity," arXiv:0810.3291 [hep-ph], JHEP 0810, 089 (2008). 
[16] T. Matsuda, "Brane inflation without slow-roll," JHEP 0703, 096 (2007) arXiv:astro-ph/0610402].

[17] G. Dvali, A. Gruzinov and M. Zaldarriaga, "A new mechanism for generating density perturbations from inflation," Phys. Rev. D 69, 023505 (2004) arXiv:astro-ph/0303591|. G. Dvali, A. Gruzinov and M. Zaldarriaga, "Cosmological perturbations from inhomogeneous reheating, freezeout, and mass domination," Phys. Rev. D 69, 083505 (2004) arXiv:astro-ph/0305548. K. Enqvist, A. Mazumdar and M. Postma, "Challenges in generating density perturbations from a fluctuating inflaton coupling," Phys. Rev. D 67, 121303 (2003) arXiv:astro-ph/0304187); F. Vernizzi, "Cosmological perturbations from varying masses and couplings," Phys. Rev. D 69, 083526 (2004) arXiv:astro-ph/0311167.

[18] K. Enqvist, A. Jokinen, A. Mazumdar, T. Multamaki and A. Vaihkonen, "Non-Gaussianity from Preheating," Phys. Rev. Lett. 94, 161301 (2005) arXiv:astro-ph/0411394; K. Enqvist, A. Jokinen, A. Mazumdar, T. Multamaki and A. Vaihkonen, "Non-gaussianity from instant and tachyonic preheating," JCAP 0503, 010 (2005) arXiv:hep-ph/0501076]; A. Jokinen and A. Mazumdar, "Very Large Primordial Non-Gaussianity from multi-field: Application to Massless Preheating," JCAP 0604, 003 (2006) arXiv:astro-ph/0512368.

[19] A. D. Linde and V. F. Mukhanov, "Nongaussian isocurvature perturbations from inflation," Phys. Rev. D 56, 535 (1997) arXiv:astro-ph/9610219]; D. H. Lyth and D. Wands, "Generating the curvature perturbation without an inflaton," Phys. Lett. B 524, 5 (2002) arXiv:hep-ph/0110002; T. Moroi and T. Takahashi, "Effects of cosmological moduli fields on cosmic microwave background," Phys. Lett. B 522, 215 (2001) [Erratum-ibid. B 539, 303 (2002)] arXiv:hep-ph/0110096].

[20] K. Dimopoulos, G. Lazarides, D. Lyth and R. Ruiz de Austri, "Curvaton dynamics," Phys. Rev. D 68, 123515 (2003) arXiv:hep-ph/0308015]; Q. G. Huang, "Curvaton with Polynomial Potential," arXiv:0808.1793 [hep-th].

[21] K. Enqvist, A. Jokinen, S. Kasuya and A. Mazumdar, "MSSM flat direction as a curvaton," Phys. Rev. D 68, 103507 (2003) arXiv:hep-ph/0303165]: K. Enqvist, 
S. Kasuya and A. Mazumdar, "Adiabatic density perturbations and matter generation from the MSSM," Phys. Rev. Lett. 90, 091302 (2003) [arXiv:hep-ph/0211147]: K. Enqvist, S. Kasuya and A. Mazumdar, "MSSM Higgses as the source of reheating and all matter," Phys. Rev. Lett. 93, 061301 (2004) [arXiv:hep-ph/0311224]. R. Allahverdi, K. Enqvist, A. Jokinen and A. Mazumdar, "Identifying the curvaton within MSSM," JCAP 0610, 007 (2006) arXiv:hep-ph/0603255.

[22] T. Matsuda, "Curvaton paradigm can accommodate multiple low inflation scales," Class. Quant. Grav. 21, L11 (2004) arXiv:hep-ph/0312058; T. Matsuda, "Topological curvatons," Phys. Rev. D 72, 123508 (2005) arXiv:hep-ph/0509063; T. Matsuda, "Hilltop Curvatons," Phys. Lett. B 659, 783 (2008) [arXiv:0712.2103 [hep-ph]].

[23] T. Matsuda, "Cosmological perturbations from an inhomogeneous phase transition," arXiv:0902.4283 [hep-ph].

[24] A. Cerioni, F. Finelli and A. Gruppuso, "Is a Dissipative Regime During Inflation in Agreement with Observations?," Phys. Rev. D 78, 021301 (2008) arXiv:0804.0163 [astro-ph]]

[25] A. A. Starobinsky, "Multicomponent de Sitter (Inflationary) Stages and the Generation of Perturbations," JETP Lett. 42 (1985) 152 [Pisma Zh. Eksp. Teor. Fiz. 42 (1985) 124]; D. S. Salopek, "Characteristics of cosmic time," Phys. Rev. D 52, 5563 (1995) arXiv:astro-ph/9506146; M. Sasaki and E. D. Stewart, "A General Analytic Formula For The Spectral Index Of The Density Perturbations Produced During Inflation," Prog. Theor. Phys. 95, 71 (1996) arXiv:astro-ph/9507001.

[26] T. Matsuda, "Evolution of curvature perturbation in generalized gravity theories," arXiv:0906.0643 [hep-th].

[27] L. M. H. Hall, I. G. Moss and A. Berera, "Scalar perturbation spectra from warm inflation," Phys. Rev. D 69, 083525 (2004) arXiv:astro-ph/0305015.

[28] M. Bastero-Gil and A. Berera, "Warm inflation model building," arXiv:0902.0521 [hep-ph]. 
[29] D. H. Lyth and Y. Rodriguez, "Non-gaussianity from the second-order cosmological perturbation," Phys. Rev. D 71, 123508 (2005) arXiv:astro-ph/0502578.

[30] T. Matsuda, "Remote Inflation: Hybrid-like inflation without hybrid-type potential," arXiv:0904.2821 [astro-ph.CO]; T. Matsuda, "Remote Inflation as hybrid-like sneutrino/MSSM inflation," arXiv:0905.4328 [hep-ph]. 